\title{
Esthesioneuroblastoma: An Update on the Massachusetts Eye and Ear Infirmary and Massachusetts General Hospital Experience with Craniofacial Resection, Proton Beam Radiation, and Chemotherapy
}

\author{
Marc W. Herr ${ }^{1,2}$ Rosh K. V. Sethi ${ }^{3} \quad$ Joshua C. Meier ${ }^{1,2}$ Kyle J. Chambers ${ }^{1}$ Aaron Remenschneider ${ }^{1}$ \\ Annie Chan ${ }^{2,4}$ William T. Curry ${ }^{2,5}$ Fred G. Barker II ${ }^{2,5}$ Daniel G. Deschler ${ }^{1} \quad$ Derrick T. Lin ${ }^{1,2}$ \\ ${ }^{1}$ Department of Otology and Laryngology, Massachusetts Eye and Ear \\ Infirmary, Boston, Massachusetts, United States \\ 2 Massachusetts Eye and Ear Infirmary/Massachusetts General Hospital \\ Address for correspondence Dr. Marc W. Herr, MD, Division of Head \\ and Neck Surgery, Department of Otology and Laryngology, \\ Massachusetts Eye and Ear Infirmary, Harvard Medical School, 243 \\ Cranial Base Center, Boston, Massachusetts, United States \\ ${ }^{3}$ Department of Otology and Laryngology, Harvard Medical School, \\ Charles Street, Boston, MA 02114, United States \\ (e-mail: marc.w.herr@gmail.com). \\ Boston, Massachusetts, United States \\ ${ }^{4}$ Department of Radiation Oncology, Massachusetts General Hospital, \\ Boston, Massachusetts, United States \\ ${ }^{5}$ Department of Neurosurgery, Massachusetts General Hospital, \\ Boston, Massachusetts, United States \\ J Neurol Surg B 2014;75:58-64.
}

\begin{abstract}
Keywords

- esthesioneuroblastoma

- craniofacial resection

- proton beam radiation

- sinonasal malignancy

Objectives To update the Massachusetts General Hospital (MGH) and Massachusetts Eye and Ear Infirmary (MEEI) experience in the management of esthesioneuroblastoma (ENB) with multimodality therapy and to reassess treatment outcomes and complications in a larger cohort with longer follow-up times.

Design A retrospective chart review.

Setting A tertiary referral center.

Participants All patients presenting with ENB and managed at the MGH and MEEI from 1997 to 2013.

Main Outcome Measures Disease-free and overall survival.

Results Twenty-two patients were identified with an average follow-up of 73 months. Ten patients presented with Kadish stage $B$ disease and 12 with stage $C$ disease. A total of six patients (27\%) developed regional metastases. Treatment for all patients included craniofacial resection (CFR) followed by proton beam irradiation with or without chemotherapy. The 5-year disease-free and overall survival rates were $86.4 \%$ and $95.2 \%$, respectively, by Kaplan-Meier analysis. Negative margins were a significant factor in disease-free survival. One patient experienced severe late-radiation toxicity. Conclusions ENB is safely and effectively treated with CFR followed by proton beam irradiation. The high incidence of regional metastases warrants strong consideration for elective neck irradiation. Proton beam radiation is associated with lower rates of severe late-radiation toxicity than conventional radiotherapy.
\end{abstract}

received

June 14, 2013

accepted after revision

July 29, 2013

published online

September 20, 2013 (c) 2014 Georg Thieme Verlag KG

Stuttgart · New York
DOI http://dx.doi.org/ 10.1055/s-0033-1356493. ISSN 2193-6331. 


\section{Introduction}

Esthesioneuroblastoma (ENB) is a relatively uncommon malignant tumor of the nasal vault believed to originate from olfactory stem cells of neural crest origin. ${ }^{1,2}$ Despite being historically labeled as a low-grade malignancy, ${ }^{3}$ ENB demonstrates a propensity for frequent recurrence following conventional treatment and a relatively high incidence of regional metastasis. ${ }^{4,5}$ Since its initial description in 1924, multiple institutions have developed treatment strategies that use various combinations of surgery, radiation therapy, and chemotherapy. ${ }^{1,2,6-14}$ Although no definitive regimen has been established, analysis of this collective experience demonstrates that multimodality therapy, particularly surgery followed by radiation, achieves the highest cure rates. ${ }^{1}$

In 2008, we published our experience treating ENB in 10 patients with craniofacial resection and adjuvant proton beam radiation with or without chemotherapy. ${ }^{15}$ Our findings reinforced the benefits of multimodality treatment and suggested that proton beam radiation might contribute to excellent outcomes while limiting severe sequelae of radiation toxicity. ${ }^{15}$ In the 5 years since this initial publication, our patient population has more than doubled. Such an increase in cohort size, along with an additional 60 months of followup time, allows for a more robust analysis of treatment outcomes and complications, as well as offering further insight into the natural history of this unique tumor. As such, this update reports and evaluates the results of our ongoing experience with a standardized multimodality treatment protocol.

\section{Materials and Methods}

After approval from the institutional review boards at Massachusetts Eye and Ear Infirmary (MEEI) and Massachusetts General Hospital (MGH), all patients diagnosed with ENB from January 1997 to January 2013 were identified by a review of records. Each patient had been evaluated by a multidisciplinary team that included a radiation oncologist, medical oncologist, otolaryngologist, pathologist, and neurosurgeon.

All patients were evaluated with magnetic resonance imaging (MRI) of the brain and skull base and a fine-cut computed tomography (CT) scan of the skull base. All medical records including imaging reports, operative reports, and chemotherapy and radiotherapy records were reviewed retrospectively. Extracted information included patient demographics, tumor stage and grade, treatment received, treatment complications, tumor recurrence, and patient survival.

The Kaplan-Meier method was used to estimate overall survival (OS), with an end point of death from any cause, and disease-free survival (DFS), with an end point of disease recurrence. Survival trends were compared by surgical margins status and tumor stage. Associations with $p<0.05$ were considered significant. All analyses were performed using Stata v.12 (StataCorp, College Station, TX, USA).

\section{Results}

\section{Patient and Tumor Characteristics}

We identified 22 patients who were treated for ENB at MEEI and MGH from 1997 to 2013. There were 11 men and 11 women in the cohort with a median age of 45.5 years (range: 11-77 years). The average follow-up time for this group was 73 months (range: $24-183$ months).

All pathologic specimens were reviewed by the Department of Pathology at MGH. The diagnosis of ENB was confirmed by a combination of histologic findings and positive immunohistochemical staining for synaptophysin, chromogranin, and $\mathrm{S} 100 .^{15}$ Tumors were generally negative for cytokeratins, desmin, vimentin, HMB45, and common leukocytic antigen. ${ }^{1,5}$

Tumors were staged using the Kadish classification ${ }^{16}$ and the TNM schema proposed by Dulguerov and Calcaterra on the basis of physical examination and radiographic findings at the time of initial presentation. ${ }^{12}$ Ten patients (45.5\%) presented with Kadish stage B disease; the remaining 12 (54.5\%) had stage $C$ disease. Using the TNM staging system, 14 patients $(63.6 \%)$ in the cohort presented with advanced (T3 or T4) tumors. Three patients (14.3\%) had regional metastases to the cervical lymph nodes at the time of presentation.

\section{Treatment}

From 1997 to 2000, the first three patients in the cohort were initially managed nonsurgically with induction chemotherapy followed by proton beam radiation, as described by Bhattacharyya and colleagues. ${ }^{2,15}$ Since there was no associated disease response in any case with induction chemotherapy, each patient was subsequently managed with craniofacial resection and adjuvant proton beam radiation. After 2000, the remaining 19 patients were managed with upfront craniofacial resection followed by adjuvant proton radiation. Concurrent chemotherapy was also administered to five patients.

\section{Surgery}

All patients underwent endoscopic intranasal biopsies as a diagnostic step. In all cases, craniofacial resection was performed by a multidisciplinary team composed of a neurosurgeon and head and neck surgeon. No patient underwent transfacial surgery alone. Endoscopic resection was initially attempted in one patient, but the procedure was aborted due to an inability to obtain complete tumor extirpation via a strictly endoscopic approach. The tumor was subsequently resected en bloc by uncomplicated craniofacial resection. Positive margins were reported in 9 of 22 patients (40.9\%). These were most commonly present at the periorbita (three patients) or posterior skull base (six patients). Selective neck dissection was performed in the three patients who presented with cervical lymph node involvement.

\section{Radiotherapy}

All patients were treated with definitive radiotherapy with curative intent. The technique for planning and delivery of proton beam therapy at MGH has been described 
previously. ${ }^{15}$ The median total dose delivered to the primary site was 66.5 cobalt gray equivalent (CGE) (range: $54-70$ ). On average, patients received 1.85 CGE per fraction over 35 fractions. A total of eight patients $(36.4 \%)$ received a combination of proton and photon radiation therapy to the neck bilaterally. In the three patients who presented with cervical lymph node metastases, the doses of therapeutic proton beam irradiation of the neck ranged from 60 to $66 \mathrm{~Gy}$. The remaining five patients were treated with bilateral elective neck irradiation (ENI): 60 CGE of proton beam radiation to the upper neck and $50 \mathrm{~Gy}$ of external beam photons to the lower neck. All patients tolerated radiotherapy without a treatment break.

\section{Chemotherapy}

As mentioned earlier, the first three patients in our series were treated with induction chemotherapy with no response. All three patients received two cycles of etoposide and cisplatin every 3 weeks. One of these patients also received an additional two cycles of carboplatin and etoposide. At the discretion of the medical oncologist, a total of five patients (22.7\%) were managed with concurrent adjuvant chemotherapy using our standard regimen of cisplatin and etoposide or carboplatin alone.

\section{Outcomes}

Local, Regional, and Distant Control

Six patients had disease recurrence an average of 73.4 months after diagnosis with a range of 13 to 145 months (- Table 1). Patient 1 was treated initially with chemotherapy, followed by craniofacial resection and proton irradiation. He recurred both regionally and distantly and was managed with surgical resection of spinal metastases and neck dissection, as well as reirradiation and chemotherapy. He is currently alive with no evidence of disease (NED) over 4.5 years later. Patient 2 developed distant metastases to the brain and spine that were managed with palliative chemotherapy. She eventually succumbed to her disease 31 months after diagnosis. Patient
20 had a recurrence in the scalp and parotid gland 13 months after diagnosis. He was treated with wide local excision, parotidectomy, and neck dissection. Twenty-eight months later, he is currently alive with disease. Patient 21 developed recurrent ENB in the neck bilaterally $\sim 2$ years after diagnosis. He was treated with bilateral neck dissections and radiation therapy to the neck and is NED 6 months after treatment.

Two patients developed multiple recurrences. Patient 3, treated with induction chemotherapy followed by craniofacial resection and proton irradiation, recurred regionally 61 months after diagnosis and was managed with a parotidectomy and neck dissection and radiation. At 145 months after diagnosis, she developed a second recurrence located at the skull base. This was treated with concomitant chemoradiation, and she is currently NED at 154 months. Patient 4, who had a gross total resection with positive microscopic margins in the sphenoid sinus, recurred locally at the edge of his frontal craniotomy bone flap 72 months after diagnosis. The tumor was managed with surgical excision and reirradiation. Metastatic lesions were subsequently discovered in his vertebrae and were treated with resection followed by concomitant chemoradiation. At 104 months, he presented with a third recurrence in his neck and ninth rib. He underwent neck dissection and received radiation therapy to the rib. Twenty months later an additional lesion developed in his sixth rib, which was also managed with radiation therapy. He currently is alive with disease 10 months later.

Overall, of the nine patients in our series with positive surgical margins, five recurred. Also noteworthy was that two of the three patients who recurred in the neck had not had ENI with their initial treatment. Additionally, all three patients who were initially managed with the induction chemotherapy protocol recurred.

\section{Survival Rates}

Kaplan-Meier survival curves were constructed for 5-year OS and DFS rates. The calculated 5-year OS and DFS rates were 95.2\% (95\% confidence interval [CI] 70.7-99.3) and 86.4\% (CI

Table 1 Tumor recurrence, treatment, and outcomes

\begin{tabular}{|c|c|c|c|c|}
\hline Patient no. & $\begin{array}{l}\text { Location of } \\
\text { recurrence }\end{array}$ & $\begin{array}{l}\text { Time to } \\
\text { recurrence, mo }\end{array}$ & Treatment & Final status \\
\hline 1 & $\begin{array}{l}\text { C-spine; left neck; } \\
\text { leptomeninges }\end{array}$ & 82 & $\begin{array}{l}\text { Rsxn C-spine metastases; } \\
\text { left ND; RT + CT }\end{array}$ & NED \\
\hline 2 & $\begin{array}{l}\text { Right frontal lobe; } \\
\text { thoracic spine }\end{array}$ & 16 & Palliative chemotherapy & DOD \\
\hline 3 & $\begin{array}{l}\text { 1. Left parotid } \\
\text { 2. Left skull base }\end{array}$ & $\begin{array}{l}61 \\
145\end{array}$ & $\begin{array}{l}\text { 1. Left parotidectomy/ND + RT } \\
\text { 2. RT }+\mathrm{CT}\end{array}$ & NED \\
\hline 4 & $\begin{array}{l}\text { 1. Temporal scalp } \\
\text { 2. Vertebrae } \\
\text { 3. Right neck; ninth rib } \\
\text { 4. Sixth rib }\end{array}$ & $\begin{array}{l}72 \\
92 \\
104 \\
124\end{array}$ & $\begin{array}{l}\text { 1. } W L E+R T \\
\text { 2. Rsxn }+ \text { RT }+C T \\
\text { 3. Right ND; RT to rib } \\
\text { 4. RT to rib }\end{array}$ & AWD \\
\hline 20 & Left scalp; left parotid & 13 & WLE; left parotidectomy/ND & AWD \\
\hline 21 & Bilateral neck & 23 & Bilateral ND + RT & NED \\
\hline
\end{tabular}

Abbreviations: AWD, alive with disease; CT, chemotherapy; DOD, died of disease; ND, neck dissection; NED, no evidence of disease; Rsxn, resection; $\mathrm{RT}$, radiation therapy; WLE, wide local excision. 
63.4-95.4), respectively (- Figs. 1 and $\mathbf{2}$ ). There was a significant improvement in 5-year DFS for patients with negative surgical margins: 92.3\% (CI 56.6-98.9) versus 77.8\% (CI 36.593.9) for positive margins (log-rank $p=0.015$ ) (-Fig. 3). There was also a trend toward improved OS with negative margins ( $100 \%$ versus $88.9 \%$ with positive margins), although this was not significant $(\log$-rank $p=0.269)$.

OS and DFS rates were also evaluated by tumor stage. There was no significant difference in 5-year OS or DFS rates between Kadish stage B and C tumors, 100\% versus $91.3 \%$ (CI 52.4-98.7) $(p=0.366)$ and $90 \%$ (CI 47.3-98.5) versus 83.3\% (CI 48.2-95.6) ( $p=0.705$ ), respectively. Results were similar when comparing tumors based on the Dulguerov staging system. The 5 -year OS rate was $100 \%$ for stage III tumors versus $88.2 \%$ (CI 41.1-98.3) for stage IV tumors $(p=0.387)$. There was also no significant difference in the 5-year DFS rate, $100 \%$ versus $77.8 \%$ (CI 36.5-93.9) for stage III and IV tumors, respectively $(p=0.786)$.

\section{Complications}

Treatment toxicity was evaluated based on the Common Terminology Criteria for Adverse Events (v.4.0) of the National Cancer Institute. Thirteen of our patients (59\%) experienced a total of 25 complications from all modalities of therapy ( - Table 2 ).

\section{Eye and Optical Axis}

Eight patients (36.4\%) had a total of 11 ocular complications. Most of these were mild to moderate in severity (grade 2) with epiphora the most common (four patients). A single case of cranial nerve VI palsy resolved spontaneously. Two cases of persistent diplopia were self-limiting and produced minimal functional limitations for the patient. A single case of severe late-radiation toxicity occurred in a patient who experienced blindness in the ipsilateral eye as a result of radiation-induced optic neuritis (grade 4) (-Table 2).

\section{Central Nervous System (CNS)}

All five reported CNS complications were experienced by the first two patients (9\%) in our cohort. Radiation brain injury

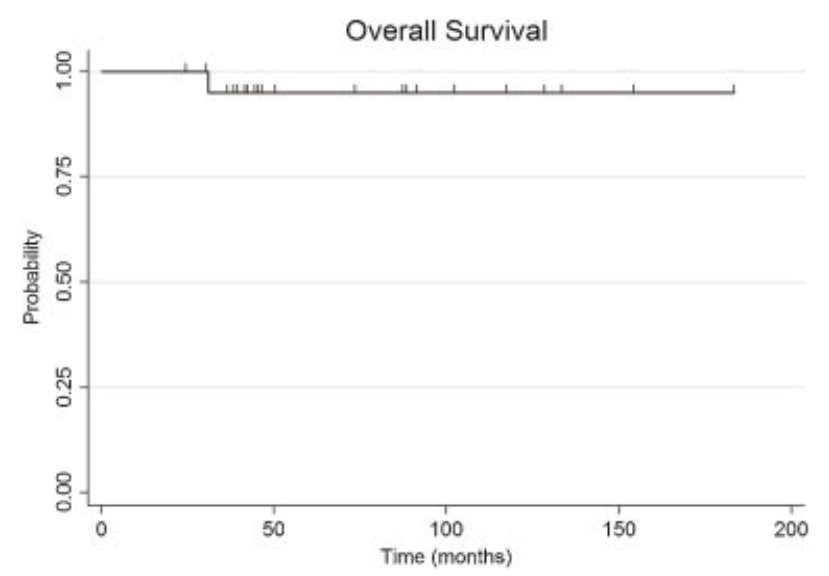

Fig. 1 The 5-year overall survival rate was 95.2\% by Kaplan-Meier analysis.

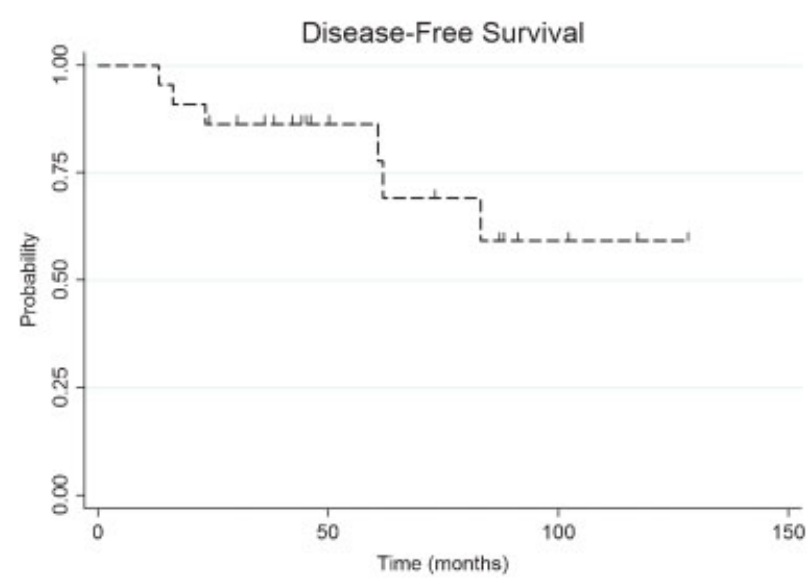

Fig. 2 The 5 -year disease free survival rate was $86.4 \%$ by Kaplan-Meier analysis.

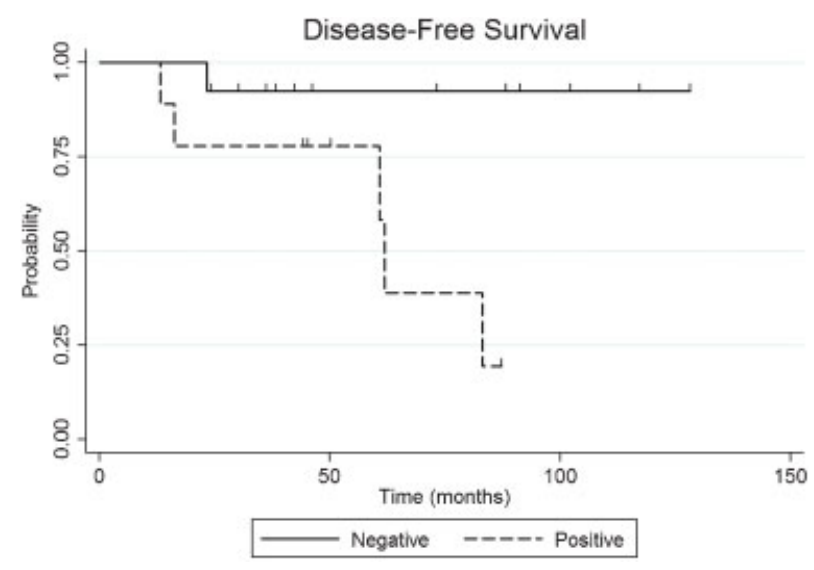

Fig. 3 The 5-year disease-free survival (DFS) Kaplan-Meier curves by surgical margin status. Five-year DFS rates were $92.3 \%$ with negative surgical margins versus $77.8 \%$ with positive margins; log-rank $p=0.015$.

was diagnosed radiographically and defined as any enhancement seen in delayed hypersensitivity on T1-weighted MRI. One patient with bifrontal lobe radiation injury experienced recurrent seizures, which are currently controlled with medication. One patient experienced a postoperative cerebrospinal fluid (CSF) leak and symptomatic pneumocephalus requiring intubation for airway diversion and an endoscopic CSF leak repair, which was successful. The second patient developed asymptomatic postoperative pneumocephalus that resolved with clamping of the lumbar drain (-Table 2 ).

\section{Wound Healing and Infectious}

Eight patients (36.4\%) had a total of nine wound complications. Four patients developed chronic sinocutaneous fistulae in the medial canthal region (grade 3). One patient was successfully repaired with a paramedian forehead flap and prosthesis placement. A second patient underwent repair with a radial forearm free (RFF) flap. She refistulized a year later and a glabellar rotational flap was used to successfully close the resulting defect. A RFF flap was used for definitive 
Table 2 Treatment complications based on Common Terminology Criteria for Adverse Events (v.4.0) of the National Cancer Institute

\begin{tabular}{|c|c|c|c|}
\hline & \multicolumn{3}{|c|}{ System organ class } \\
\hline Grade & $\begin{array}{l}\text { Eye and optical axis } \\
\text { (no. of patients) }\end{array}$ & $\begin{array}{l}\text { Central nervous system } \\
\text { (no. of patients) }\end{array}$ & $\begin{array}{l}\text { Wound healing and } \\
\text { infectious (no. of patients) }\end{array}$ \\
\hline \multicolumn{4}{|l|}{1} \\
\hline 2 & $\begin{array}{l}\text { - Epiphora (4) } \\
\text { - Diplopia (2) } \\
\text { - Globe ptosis (1) } \\
\text { - Cranial nerve VI palsy (1) } \\
\text { - Retinopathy (2) }\end{array}$ & $\begin{array}{l}\text { - Radiation brain injury (2) } \\
\text { - Preumocephalus (1) }\end{array}$ & $\begin{array}{l}\text { - Wound infection (1) } \\
\text { - Orbital cellulitis (1) }\end{array}$ \\
\hline 3 & & $\begin{array}{l}\text { - CSF leak (1) } \\
\text { - Pneumocephalus (1) }\end{array}$ & $\begin{array}{l}\text { - Osteomyelitis of bone flap (1) } \\
\text { - ORN of skull base (1) } \\
\text { - Sinocutaneous fistula (4) } \\
\text { - Frontal sinus abscess (1) }\end{array}$ \\
\hline 4 & - Unilateral blindness (1) & & \\
\hline Total, \% & 36 & 9 & 36 \\
\hline
\end{tabular}

Abbreviations: CSF, cerebrospinal fluid; ORN, osteoradionecrosis.

repair of a sinocutaneous fistula in the setting of osteoradionecrosis of the skull base in a third patient. The final patient succumbed to progressive disease prior to fistula repair.

Several infectious complications were also reported. A postoperative wound infection (grade 2) and an episode of orbital cellulitis (grade 2) were successfully addressed with intravenous antibiotics. One patient developed osteomyelitis of the frontal bone flap (grade 3 ) 2 years postoperatively, but this also resolved with intravenous antibiotics. Finally, antibiotics and surgical debridement were required to address a frontal sinus abscess (grade 3 ) that developed in another patient.

\section{Discussion}

With an average follow-up $>6$ years, our 5-year DFS rates (86.4\%) and OS rates (95.2\%) compare extremely favorably with the literature, which reports DFS rates ranging from 33\% to $87 \%$ and OS rates from $60 \%$ to $76 \%{ }^{1,7,8,10,13,17,18}$ This is undoubtedly most attributable to the use of a consistent multimodality treatment algorithm, which is supported in the literature. Organizing the treatment, however, remains somewhat controversial. In $>40$ years of experience treating ENB with preoperative radiation therapy with or without chemotherapy followed by craniofacial resection, the group at the University of Virginia has achieved an impressive 15-year DFS rate of 82.6\%. ${ }^{7,8}$ Nevertheless, the meta-analysis by Dulguerov and colleagues demonstrates that surgery followed by adjuvant radiation is the most frequently used approach and also produces the highest OS rates: $65 \%$ at 5 years. ${ }^{1}$ Our series reinforces these findings and shows excellent outcomes with upfront craniofacial resection followed by adjuvant radiation therapy with or without chemotherapy. Our experience also demonstrates the importance of negative surgical margins in local control and highlights the benefits of craniofacial resection, which permits en bloc extirpation of the tumor as well as better assessment of margin status and intracranial extension. ${ }^{1}$
This, too, is consistent with the reported experience of several institutions that have demonstrated superior local control and improved OS with craniofacial resection over transfacial approaches. ${ }^{7,11,12,17}$

\section{Locoregional Disease and Recurrence}

A collective review of the contemporary ENB literature reveals a high rate of cervical nodal metastases, ranging from $17 \%$ to $33 \%{ }^{4,5,14,19-22}$ Furthermore, cervical lymph node involvement has been established as a negative prognostic indicator. Koka and colleagues reported a 0\% 2-year survival rate for patients with regional metastases. ${ }^{19}$ Dulguerov's meta-analysis found a significant difference in survival, noting that only $29 \%$ of node-positive patients were treated successfully, compared with $64 \%$ of N0 patients. ${ }^{1}$ In their series of 27 patients, Resto et al described cervical lymph node involvement in a total of 9 patients (33\%). ${ }^{14}$ Statistical analysis showed nodal metastasis to be the only significant predictor of survival. Seven patients without evidence of cervical nodal metastases at the time of definitive treatment received elective radiation therapy to the ipsilateral neck. In this group, only one patient (who also had positive surgical margins) went on to develop cervical nodal disease 3 years after treatment. ${ }^{14}$

In our series, a total of 6 patients (27\%) developed metastatic disease in the cervical lymph nodes: three at presentation and three with regional recurrence. Notably, in the three patients with regional recurrence, two had not received elective radiation to the neck. One patient subsequently developed additional distant metastases and is currently alive with disease. The remaining five patients are alive with no evidence of disease. The reason for this is undoubtedly multifactorial but likely due, at least in part, to the aggressive identification and management of the disease process. Given the high rate of documented regional involvement, as well as the prognostic significance described in the literature, we now routinely perform bilateral ENI in all ENB patients. 
Future analysis of this growing subset of patients will provide a more definitive assessment on outcomes using this approach.

\section{Proton Beam Radiation, Outcomes, and Late Toxicity}

Standard radiotherapy for tumors of the skull base typically involves external-beam photons and a three-field technique, with doses ranging from 55 to 65 Gy. ${ }^{1,15}$ The associated radiation toxicity and resulting complications, especially along the optical axis, can be a significant problem. Indeed, the literature reports severe ocular radiation injury leading to a poor or nonfunctioning eye at rates ranging from $8 \%$ to $24 \%{ }^{10,12,15,23}$ Unlike photons, which deliver their highest radiation dose at the skin followed by a continuously decreasing dose with increasing depth of penetration, protons deposit nearly all of their energy at the point of greatest penetration. This phenomenon is known as the Bragg peak. The exact depth to which protons penetrate depends on the energy of the proton beam, which can be precisely controlled to place the Bragg peak within the targeted tissues. ${ }^{24,25}$ Furthermore, because most protons are absorbed at this point without an exit dose, normal tissues beyond the target receive very little or no radiation. ${ }^{24}$

Theoretically, these properties should allow for maximal radiation doses to be focused on the tumor, thus improving local control while sparing adjacent uninvolved tissues, thereby decreasing the incidence and severity of acute and late toxic effects. ${ }^{15,24,25}$ With sinonasal malignancies, this offers the additional advantage of sparing critical adjacent structures, such as the globe, optic nerves, optic chiasm, and brain. ${ }^{15}$ In spite of this, the use of proton beam therapy as a component of multimodality treatment for ENB has not been examined extensively in the literature. Only a handful of studies have documented their experience using proton beam radiation in the treatment of sinonasal and skull base malignancies, including ENB and neuroendocrine carcinoma, as well as adenoid cystic carcinoma. ${ }^{2,9,15,26,27}$

In general, the authors reported excellent local control and survival rates while toxic effects were minimized. In their series of nine patients treated with induction chemotherapy followed proton beam radiation, Bhattacharyya et al noted only a single ocular complication, which resolved spontaneously. ${ }^{2}$ However, the median follow-up was only 20.5 months. With a longer follow-up of 40 months, Nishimura and colleagues reported no toxic effects greater than grade $2 .{ }^{9}$ Fitzek et al experienced no significant ocular complications, although four patients did experience grade 2 or 3 radiation injury to the frontal lobes. ${ }^{26}$ Using proton beam radiation as monotherapy or as adjuvant treatment following surgical resection, Pommier et al achieved an encouraging 5-year local control rate of $93 \%$ for patients with adenoid cystic carcinoma of the skull base. ${ }^{27}$ The median dose delivered to the primary site was 76.4 CGE. Subsequent ocular toxicities were not insignificant; one patient developed chronic grade 4 retinopathy and three patients had chronic grade 3 adverse effects requiring surgical intervention. ${ }^{27}$ Furthermore, 10 patients developed grade 3 radiation-induced brain injury manifested by seizures or memory loss, and one patient died from the toxic effects of radiation-induced brain injury (grade 5) at 61 months after radiation treatment. Another patient succumbed to infectious meningitis secondary to a persistent CSF leak at 9 months following radiation therapy. ${ }^{27}$

All patients in our series were treated with proton beam radiation to the primary site. Even though the median radiation dose to the tumor was near maximal at 66.5 CGE, there were no breaks in treatment as a result of acute toxicities. However, with an average follow-up of 73 months, 13 patients (59\%) did experience several mild to severe complications due to late-radiation toxicity. This included a case of ipsilateral blindness resulting from radiation-induced optic neuritis. Because this patient presented with intracranial extension of her tumor, had positive surgical margins at the skull base, and was treated with 70 CGE plus concomitant cisplatin and etoposide, she was undoubtedly at increased risk for treatment morbidities. In several other cases, surgical intervention was required to resolve adverse radiation effects, including four persistent sinocutaneous fistulas and three infectious complications at the anterior skull base.

Thus although the use of proton beam radiation may have minimized acute radiation toxicity in our patients, extended follow-up reveals the development of several ocular and wound complications resulting from late-radiation toxicity. Nevertheless, many of these were mild to moderate in severity and have either resolved or been corrected with appropriate treatment. Furthermore, despite an unfortunate case of blindness (4.5\%), our rate of severe ocular injury remains significantly less than those resulting from standard radiation protocols.

\section{Conclusion}

ENB is a unique tumor of the skull base with a predilection for late recurrence, as well as regional and distant metastasis. Over the past 16 years, we have used a combined treatment protocol consisting of craniofacial resection followed by proton beam radiation and demonstrated excellent survival outcomes. When placed in this context, and considering that severe complications from radiation toxicity are less than those reported for standard radiotherapy, proton beam radiation deserves consideration as a worthwhile alternative to more traditional radiation treatment regimens for ENB. Additional experience with more patients and longer follow-up should provide more compelling information regarding its proper use.

\section{Acknowledgments}

The authors would like to acknowledge Dr. Anthony C. Nichols, MD, for his work on the first review of this patient cohort and for his contributions to cancer research.

\section{Note}

This work was presented as an oral presentation at the 23rd Annual North American Skull Base Society Meeting in Miami, Florida, on February 15, 2013. 
64 Esthesioneuroblastoma: MEEI and MGH Update Herr et al.

\section{Disclosures}

The authors have no conflicts of interest to report.

\section{References}

1 Dulguerov P, Allal AS, Calcaterra TC. Esthesioneuroblastoma: a meta-analysis and review. Lancet Oncol 2001;2(11):683-690

2 Bhattacharyya N, Thornton AF, Joseph MP, Goodman ML, Amrein PC. Successful treatment of esthesioneuroblastoma and neuroendocrine carcinoma with combined chemotherapy and proton radiation. Results in 9 cases. Arch Otolaryngol Head Neck Surg 1997;123(1):34-40

3 Bailey BJ, Barton S. Olfactory neuroblastoma. Management and prognosis. Arch Otolaryngol 1975;101(1):1-5

4 Beitler JJ, Fass DE, Brenner HA, et al. Esthesioneuroblastoma: is there a role for elective neck treatment? Head Neck 1991;13 (4):321-326

5 Rinaldo A, Ferlito A, Shaha AR, Wei WI, Lund VJ. Esthesioneuroblastoma and cervical lymph node metastases: clinical and therapeutic implications. Acta Otolaryngol 2002;122(2):215-221

6 Berger L, Luc R, Richard D. L'esthésioneuroépithéliome olfactif. Bull Assoc Fr Etud Cancer 1924;13:410-421

7 Levine PA, Gallagher R, Cantrell RW. Esthesioneuroblastoma: reflections of a 21-year experience. Laryngoscope 1999; 109(10):1539-1543

8 Loy AH, Reibel JF, Read PW, et al. Esthesioneuroblastoma: continued follow-up of a single institution's experience. Arch Otolaryngol Head Neck Surg 2006;132(2):134-138

9 Nishimura H, Ogino T, Kawashima M, et al. Proton-beam therapy for olfactory neuroblastoma. Int J Radiat Oncol Biol Phys 2007; 68(3):758-762

10 Simon JH, Zhen W, McCulloch TM, et al. Esthesioneuroblastoma: the University of Iowa experience 1978-1998. Laryngoscope 2001;111(3):488-493

11 Dias FL, Sa GM, Lima RA, et al. Patterns of failure and outcome in esthesioneuroblastoma. Arch Otolaryngol Head Neck Surg 2003; 129(11):1186-1192

12 Dulguerov P, Calcaterra T. Esthesioneuroblastoma: the UCLA experience 1970-1990. Laryngoscope 1992;102(8):843-849

13 Constantinidis J, Steinhart H, Koch M, et al. Olfactory neuroblastoma: the University of Erlangen-Nuremberg experience 19752000. Otolaryngol Head Neck Surg 2004;130(5):567-574
14 Resto VA, Eisele DW, Forastiere A, Zahurak M, Lee DJ, Westra WH. Esthesioneuroblastoma: the Johns Hopkins experience. Head Neck 2000;22(6):550-558

15 Nichols AC, Chan AW, Curry WT, Barker FG, Deschler DG, Lin DT. Esthesioneuroblastoma: the Massachusetts Eye and Ear Infirmary and Massachusetts General Hospital experience with craniofacial resection, proton beam radiation, and chemotherapy. Skull Base 2008;18(5):327-337

16 Kadish S, Goodman M, Wang CC. Olfactory neuroblastoma. A clinical analysis of 17 cases. Cancer 1976;37(3):1571-1576

17 Lund VJ, Howard D, Wei W, Spittle M. Olfactory neuroblastoma: past, present, and future? Laryngoscope 2003;113(3):502-507

18 Argiris A, Dutra J, Tseke P, Haines K. Esthesioneuroblastoma: the Northwestern University experience. Laryngoscope 2003; 113(1):155-160

19 Koka VN, Julieron M, Bourhis J, et al. Aesthesioneuroblastoma. J Laryngol Otol 1998;112(7):628-633

20 Irish J, Dasgupta R, Freeman J, et al. Outcome and analysis of the surgical management of esthesioneuroblastoma. J Otolaryngol 1997;26(1):1-7

21 Morita A, Ebersold MJ, Olsen KD, Foote RL, Lewis JE, Quast LM. Esthesioneuroblastoma: prognosis and management. Neurosurgery 1993;32(5):706-714; discussion 714-715

22 Shaari CM, Catalano PJ, Sen C, Post K. Central nervous system metastases from esthesioneuroblastoma. Otolaryngol Head Neck Surg 1996;114(6):808-812

23 Hwang SK, Paek SH, Kim DG, Jeon YK, Chi JG, Jung HW. Olfactory neuroblastomas: survival rate and prognostic factor. J Neurooncol 2002;59(3):217-226

24 Chan AW, Liebsch NJ. Proton radiation therapy for head and neck cancer. J Surg Oncol 2008;97(8):697-700

25 van de Water TA, Bijl HP, Schilstra C, Pijls-Johannesma M, Langendijk JA. The potential benefit of radiotherapy with protons in head and neck cancer with respect to normal tissue sparing: a systematic review of literature. Oncologist 2011;16(3):366-377

26 Fitzek MM, Thornton AF, Varvares M, et al. Neuroendocrine tumors of the sinonasal tract. Results of a prospective study incorporating chemotherapy, surgery, and combined proton-photon radiotherapy. Cancer 2002;94(10):2623-2634

27 Pommier P, Liebsch NJ, Deschler DG, et al. Proton beam radiation therapy for skull base adenoid cystic carcinoma. Arch Otolaryngol Head Neck Surg 2006;132(11):1242-1249 